\title{
SEMISYNTHETIC CEPHALOSPORINS WITH ANTIFUNGAL ACTIVITY: LABORATORY STUDIES ON 2-PYRIDINETHIOL 1-OXIDE CEPHALOSPORINS
}

\author{
Joseph V. Uri, Paul Actor, Lillian Phillips and Jerry A. Weisbach \\ Research and Development Division, Smith Kline \& French Laboratories \\ Philadelphia, Pennsylvania 19101, U. S. A.
}

(Received for publication March 27, 1978)

\begin{abstract}
Cephalosporins are chemotherapeutic agents whose spectrum and use are limited to antibacterial activity. Therefore, it was of interest to find several new semisynthetic cephalosporins which possess in vitro antifungal activity against Trichophyton mentagrophytes, Candida albicans and certain other yeasts. Five new cephalosporins containing the 2-pyridinethiol 1oxide grouping were examined. Four with this heterocyclic moiety in the 3-position were found to have activity. One with the grouping in the 7-acyl-side chain was inactive. The degree of the antifungal activity was influenced by the substituent at the 7-position. The cephalosporin with a $p$-hydroxyphenylglycyl side chain was the most potent against the fungal strains studied. Despite this in vitro antifungal activity, none of the compounds protected mice against a systemic Candida albicans infection. All of these cephalosporins had broad spectrum in vitro and in vivo antibacterial activity.
\end{abstract}

The composition and function of fungal cell wall are different from those of bacteria, therefore, it is of interest that several semisynthetic cephalosporins which affect bacterial cell wall synthesis were found to have in vitro activity against Trichophyton mentagrophytes, Candida albicans, Saccharomyces cerevisiae, Torulopsis glabrata, Pichia pinus and a Kloeckeria sp. Four such semisynthetic cephalosporins are described in this study. All $(\mathbf{1} \sim \mathbf{4})$ have a 2-pyridinethiol 1-oxide moiety (Omadine) at the 3'position. Omadine is a disinfectant and antifungal agent used in ointment, soaps and toiletries ${ }^{2,7}$. GoTTSTEIN and co-workers ${ }^{4}$ have described N-benzyldithiocarbamoyl-acetamidocephalosporanic acid in which the antifungal activity is a property of the intact cephalosporin molecule, although the side chain itself had antifungal activity. Included in this study is a semisynthetic cephalosporin (5) in which the omadine moiety is attached to the 7-acylamino side chain. This analog possesses no antifungal activity.

\section{Materials and Methods}

\section{Compounds}

The chemical structures of the five cephalosporins included in this study are shown in Fig. 1. The four cephalosporins with a 2-pyridinethiol 1-oxide substituent at the 3'-position (1 $\sim 4)$ have a thienylacetyl, mandeloyl, phenylglycyl or p-hydroxyphenylglycyl substituent on the 7-amino group. Compound 5 with the 2-pyridinethiol 1-oxide acetamido group at the 7-position has a 1-methyltetrazolethiomethyl group at the 3-position. The cephalosporins used in the study were synthesized at Smith Kline \& French Laboratories. The antifungal controls, 2-pyridinethiol 1-oxide, nystatin, amphotericin $\mathrm{B}$, griseofulvin and tolnaftate, and the antibacterial controls, cefazolin and cephalexin were commercially-available preparations.

\section{Organisms}

Trichophyton mentagrophytes 1258, Candida albicans 759 and the yeast strains of Saccharomyces cerevisiae, Torulopsis glabrata, Pichia pinus and Kloeckeria, sp. are strains regularly used in our laboratories. The in vitro antimicrobial activities were determined by disc agar diffusion or by serial two-fold agar dilution methods using SABOURAUD's glucose agar (SAB), Trypticase soy agar (TSA) or Penassay 
seed agar (PSA). The C. albicans and bacterial plates were incubated overnight at $37^{\circ} \mathrm{C}$ and the T. mentagrophytes plates for 3 days at $30^{\circ} \mathrm{C}$.

In vivo protection data were obtained using albino male mice weighing $18 \sim 20 \mathrm{~g}$ infected with $C$. albicans, E. coli or $K$. pneumoniae. In the case of $C$. albicans 759 , mice were infected intravenously $(0.2 \mathrm{ml})$ with a saline suspension of an 18-hour SABOURAUD's glucose agar culture (approximately $2 \times 10^{8}$ cells/mouse). The mice were treated subcutaneously, one and five hours post-infection, and the $\mathrm{ED}_{50}$ values, expressed as total dose, were calculated by the method of LitCHFIELD and WILCOXON ${ }^{6)}$. The bacterial infection-protection tests and the pharmacokinetic studies (serum levels, urinary excretion) were performed using procedures described previous$1 y^{1,11}$.

\section{Results}

\section{In Vitro Studies}

The in vitro activities against $T$. mentagrophytes 1258 for the cephalosporins and several controls are summarized in Table 1. Activities
Fig. 1. Structures of 2-pyridinethiol 1-oxide cephalosporins<smiles>CCC1=C(C(=O)O)N2C(=O)C(CC)C2SC1</smiles>

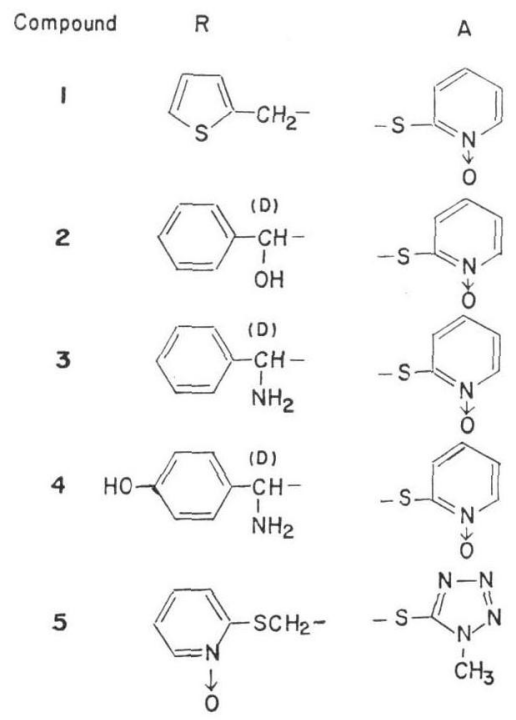
against C. albicans 759 are shown in Table 2, and against the four yeast strains in Table 3.

Of the four cephalosporins with the 2-pyridinethiol 1-oxide substituent at the $3^{\prime}$-position, those with a phenylglycine side chain at the 7-position $(3,4)$ exhibited the best activity against $T$. mentagrophytes. The mandeloyl derivative (2) was less active and the thienylacetyl derivative (1) essentially inactive against this organism. Cephalosporin 5 which contains the 2-pyridinethiol 1-oxide moiety in the acyl side chain is inactive against $T$. mentagrophytes. None of the cephalosporins were as active in vitro as the three antifungal controls (omadine, griseofulvin, tolnaftate).

The activity against Candida albicans (Table 2) follows the same order of potency observed with $T$. mentagrophytes, however, the inhibition zones are consistently larger. In many cases double inhibition zones were observed with a well-defined inner zone surrounded by a hazy outer zone. Again compound 5 was virtually without activity. In this case, the activities exhibited by cephalosporins $1 \sim 4$ compare more favorably with those of the antifungal controls.

Against the four yeast strains, all four cephalosporins with the 2-pyridinethiol 1-oxide side chain at the 3 -position possess basically the same pattern of activity as against C. albicans (Table 3). The strains showed different degrees of sensitivity; Pichia pinus was the most sensitive and the Kloeckeria sp. the least sensitive. Compound 4 was the most potent against all strains on all three media. Compound 5 was inactive. Omadine proved to be more potent against these yeast strains than either nystatin or amphotericin B.

Compound $\mathbf{4}$ also was compared with omadine, amphotericin B, cefazolin and cephalexin in an agar dilution assay using C. albicans. The MIC value for 4 was only one dilution less than that of omadine (12.5 versus $6.3 \mu \mathrm{g} / \mathrm{ml})$. As expected, amphotericin B was more active $(0.2 \mu \mathrm{g} / \mathrm{ml})$ and the two known 
Table 1. In vitro activity against Trichophyton mentagrophytes $\$ 1258$ of 2-pyridinethiol 1-oxide cephalosporins and of controls on three media

\begin{tabular}{|c|c|c|c|c|}
\hline \multirow{2}{*}{$\begin{array}{c}\text { Test } \\
\text { compound }\end{array}$} & \multirow{2}{*}{$\mu \mathrm{g} / \mathrm{disc}$} & \multicolumn{3}{|c|}{$\begin{array}{c}\text { Diameter of inhibition } \\
\text { zone }(\mathrm{mm})\end{array}$} \\
\hline & & $\mathrm{SAB}^{1}$ & $\mathrm{TSA}^{2}$ & $\mathrm{PSA}^{3}$ \\
\hline 1 & $\begin{array}{c}10 \\
1 \\
0.1\end{array}$ & $\begin{array}{l} \pm \\
0 \\
0\end{array}$ & $\begin{array}{l}0 \\
0 \\
0\end{array}$ & $\begin{array}{c}(10) \\
0 \\
0\end{array}$ \\
\hline 2 & $\begin{array}{l}10 \\
1 \\
0.1\end{array}$ & $\begin{array}{c}8(16) \\
0 \\
0\end{array}$ & $\begin{array}{c} \pm \\
0 \\
0\end{array}$ & $\begin{array}{c}9(17) \\
0 \\
0\end{array}$ \\
\hline 3 & $\begin{array}{l}10 \\
1 \\
0.1\end{array}$ & $\begin{array}{l}19 \\
\pm \\
0\end{array}$ & $\begin{array}{r}16 \\
0 \\
0\end{array}$ & $\begin{array}{c}26 \\
(11) \\
0\end{array}$ \\
\hline 4 & $\begin{array}{l}10 \\
1 \\
0.1\end{array}$ & $\begin{array}{l}23 \\
\pm \\
0\end{array}$ & $\begin{array}{c}11(21) \\
0 \\
0\end{array}$ & $\begin{array}{r}26 \\
11 \\
0\end{array}$ \\
\hline 5 & $\begin{array}{l}10 \\
1 \\
0.1\end{array}$ & $\begin{array}{l} \pm \\
0 \\
0\end{array}$ & $\begin{array}{l}0 \\
0 \\
0\end{array}$ & $\begin{array}{l}\text { N.D. } \\
\text { N.D. } \\
\text { N.D. }\end{array}$ \\
\hline $\begin{array}{l}\text { Omadine } \\
\text { sodium }\end{array}$ & $\begin{array}{l}10 \\
1 \\
0.1\end{array}$ & $\begin{array}{r}44 \\
21 \\
0\end{array}$ & $\begin{array}{c}24(39) \\
10 \\
0\end{array}$ & $\begin{array}{c}43 \\
14(19) \\
\pm\end{array}$ \\
\hline Griseofulvin & $\begin{array}{l}10 \\
1 \\
0.1\end{array}$ & $\begin{array}{r}30 \\
10 \\
0\end{array}$ & $\begin{array}{r}38 \\
20 \\
0\end{array}$ & $\begin{array}{r}33 \\
16 \\
0\end{array}$ \\
\hline Tolnaftate & $\begin{array}{l}10 \\
1 \\
0.1\end{array}$ & $\begin{array}{l}38 \\
38 \\
29\end{array}$ & $\begin{array}{l}39 \\
39 \\
32\end{array}$ & $\begin{array}{l}32 \\
34 \\
29\end{array}$ \\
\hline
\end{tabular}

1. Sabouraud's glucose agar. 2. Trypticase soy agar. 3. Penassay seed agar.

( ) Inhibition with hazy edges. \pm Very small inhibition zone. N.D. Not done
Table 2. In vitro activity against Candida albicans \#759 of 2-pyridinethiol 1-oxide cephalosporins and of controls on three media

\begin{tabular}{|c|c|c|c|c|}
\hline \multirow{2}{*}{$\begin{array}{c}\text { Test } \\
\text { compound }\end{array}$} & \multirow{2}{*}{$\mu \mathrm{g} /$ disc } & \multicolumn{3}{|c|}{$\begin{array}{l}\text { Diameter of inhibition } \\
\text { zone (mm) }\end{array}$} \\
\hline & & $\mathrm{SAB}^{1}$ & $\mathrm{TSA}^{2}$ & $\mathrm{PSA}^{3}$ \\
\hline 1 & $\begin{array}{l}10 \\
1 \\
0.1\end{array}$ & $\begin{array}{r}19 \\
0 \\
0\end{array}$ & $\begin{array}{c}(10) \\
0 \\
0\end{array}$ & $\begin{array}{c}(20) \\
0 \\
0\end{array}$ \\
\hline 2 & $\begin{array}{c}10 \\
1 \\
0.1\end{array}$ & $\begin{array}{c}20(26) \\
(9) \\
0\end{array}$ & $\begin{array}{c}(16) \\
0 \\
0\end{array}$ & $\begin{array}{c}14(24) \\
\pm \\
0\end{array}$ \\
\hline 3 & $\begin{array}{l}10 \\
1 \\
0.1\end{array}$ & $\begin{array}{c}24 \\
10 \\
\pm\end{array}$ & $\begin{array}{r}20 \\
15 \\
0\end{array}$ & $\begin{array}{c}24(26) \\
(18) \\
\pm\end{array}$ \\
\hline 4 & $\begin{array}{l}10 \\
1 \\
0.1\end{array}$ & $\begin{array}{c}26(31) \\
9(13) \\
0\end{array}$ & $\begin{array}{c}22(31) \\
14 \\
0\end{array}$ & $\begin{array}{c}20(29) \\
19 \\
0\end{array}$ \\
\hline 5 & $\begin{array}{l}10 \\
1 \\
0.1\end{array}$ & $\begin{array}{l} \pm \\
0 \\
0\end{array}$ & $\begin{array}{l}0 \\
0 \\
0\end{array}$ & $\begin{array}{l}\text { N.D. } \\
\text { N.D. } \\
\text { N.D. }\end{array}$ \\
\hline $\begin{array}{l}\text { Omadine } \\
\text { sodium }\end{array}$ & $\begin{array}{l}10 \\
1 \\
0.1\end{array}$ & $\begin{array}{c}18(32) \\
12(29) \\
(15)\end{array}$ & $\begin{array}{c}19(39) \\
(27) \\
(12)\end{array}$ & $\begin{array}{c}20(39) \\
(26) \\
(18)\end{array}$ \\
\hline Nystatin & $\begin{array}{l}10 \\
1 \\
0.1\end{array}$ & $\begin{array}{r}22 \\
8 \\
0\end{array}$ & $\begin{array}{r}25 \\
9 \\
0\end{array}$ & $\begin{array}{c}12(26) \\
(8) \\
0\end{array}$ \\
\hline $\begin{array}{l}\text { Amphoteri- } \\
\operatorname{cin} B\end{array}$ & $\begin{array}{l}10 \\
1 \\
0.1\end{array}$ & $\begin{array}{r}20 \\
14 \\
9\end{array}$ & $\begin{array}{l}25 \\
21 \\
18\end{array}$ & $\begin{array}{l}18 \\
14 \\
10\end{array}$ \\
\hline
\end{tabular}

1. Sabouraud's glucose agar. 2. Trypticase soy agar. 3. Penassay seed agar.

( ) Inhibition with hazy edges. \pm Very small inhibition zone. N.D. Not done.

antibacterial cephalosporins were inactive against C.albicans. Data in Table 4 show the in vitro activities of the five cephalosporins and those of omadine, cefazolin and cephalexin against 15 standard strains of bacteria ${ }^{11}$. All of the test cephalosporins have broad spectrum antibacterial activities comparable to those of omadine, cefazolin and cephalexin.

\section{In Vivo Studies}

Although all four cephalosporins containing the 2-pyridinethiol 1-oxide at the 3'-position were found to possess in vitro antifungal activity, none of them protected or prolonged survival time of mice experimentally infected with C. albicans $\left(\mathrm{ED}_{50} \geqq 200 \mathrm{mg} / \mathrm{kg}\right.$ ), whereas, amphotericin B gave good protective activity $\left(E D_{50}=0.08 \mathrm{mg} / \mathrm{kg}\right)$. In contrast to this finding, all these cephalosporins protected mice against experimental systemic infections with Escherichia coli and Klebsiella pneumoniae(Table 5). The subcutaneous $\mathrm{ED}_{50}$ values are best for $\mathbf{5}$ and $\mathbf{4}$ and the poorest for $\mathbf{1}$. Oral administration of compound 4 produced some protection in both infections. Omadine, when injected subcutaneously, was not protective in $E$. coli infection.

The pharmacokinetic behavior of these cephalosporins was in agreement with the mouse protection 
Table 3. In vitro anti-yeast activity of 2-pyridinethiol 1-oxide cephalosporins and of controls on three media.

Figures are diameters $(\mathrm{mm})$ of inhibition zones

\begin{tabular}{|c|c|c|c|c|c|c|c|c|c|c|c|c|c|}
\hline \multirow{2}{*}{$\begin{array}{l}\text { Test } \\
\text { compound }\end{array}$} & \multirow{2}{*}{$\mu \mathrm{g} / \mathrm{disc}$} & \multicolumn{3}{|c|}{$\begin{array}{c}\text { Saccharomyces } \\
\text { cerevisiae }\end{array}$} & \multicolumn{3}{|c|}{$\begin{array}{l}\text { Torulopsis } \\
\text { glabrata }\end{array}$} & \multicolumn{3}{|c|}{ Pichia pinus } & \multicolumn{3}{|c|}{$\begin{array}{l}\text { Kloeckeria } \\
\text { sp. }\end{array}$} \\
\hline & & $\mathrm{SAB}^{1}$ & TSA $^{2}$ & $\mathrm{PSA}^{3}$ & $\mathrm{SAB}$ & TSA & PSA & SAB & TSA & PSA & $\mathrm{SAB}$ & TSA & PSA \\
\hline 1 & $\begin{array}{c}10 \\
1 \\
0.1\end{array}$ & $\begin{array}{r}15 \\
12 \\
0\end{array}$ & $\begin{array}{c}10(19) \\
0 \\
0\end{array}$ & $\begin{array}{c}10(19) \\
(8) \\
0\end{array}$ & $\begin{array}{c}(20) \\
(12) \\
0\end{array}$ & $\begin{array}{r}15 \\
0 \\
0\end{array}$ & $\begin{array}{c}16 \\
\pm \\
0\end{array}$ & $\begin{array}{c}22(27) \\
12 \\
0\end{array}$ & $\begin{array}{l}\text { N.G. } \\
\text { N.G. } \\
\text { N.G. }\end{array}$ & $\begin{array}{c}12(22) \\
0 \\
0\end{array}$ & $\begin{array}{r}12 \\
9 \\
0\end{array}$ & $\begin{array}{l}7 \\
0 \\
0\end{array}$ & $\begin{array}{l}8 \\
0 \\
0\end{array}$ \\
\hline 2 & $\begin{array}{l}10 \\
1 \\
0.1\end{array}$ & $\begin{array}{r}16 \\
8 \\
0\end{array}$ & $\begin{array}{c}(18) \\
9 \\
0\end{array}$ & $\begin{array}{c}10(19) \\
8 \\
0\end{array}$ & $\begin{array}{c}(20) \\
(11) \\
0\end{array}$ & $\begin{array}{c}(18) \\
0 \\
0\end{array}$ & $\begin{array}{c}(24) \\
(11) \\
0\end{array}$ & $\begin{array}{r}24 \\
13 \\
0\end{array}$ & $\begin{array}{l}\text { N.G. } \\
\text { N.G. } \\
\text { N.G. }\end{array}$ & $\begin{array}{c}(35) \\
\pm \\
0\end{array}$ & $\begin{array}{r}13 \\
8 \\
0\end{array}$ & $\begin{array}{c}(8) \\
0 \\
0\end{array}$ & $\begin{array}{c}(8) \\
0 \\
0\end{array}$ \\
\hline 3 & $\begin{array}{l}10 \\
1 \\
0.1\end{array}$ & $\begin{array}{r}20 \\
11 \\
0\end{array}$ & $\begin{array}{c}(19) \\
(9) \\
0\end{array}$ & $\begin{array}{r}16 \\
8 \\
0\end{array}$ & $\begin{array}{c}(19) \\
(12) \\
0\end{array}$ & $\begin{array}{c}(30) \\
(16) \\
0\end{array}$ & $\begin{array}{c}11(29) \\
(17) \\
0\end{array}$ & $\begin{array}{c}22(35) \\
16 \\
0\end{array}$ & $\begin{array}{l}\text { N.G. } \\
\text { N.G. } \\
\text { N.G. }\end{array}$ & $\begin{array}{r}50 \\
22 \\
0\end{array}$ & $\begin{array}{r}18 \\
12 \\
0\end{array}$ & $\begin{array}{c}16 \\
\pm \\
0\end{array}$ & $\begin{array}{r}13 \\
0 \\
0\end{array}$ \\
\hline 4 & $\begin{array}{l}10 \\
1 \\
0.1\end{array}$ & $\begin{array}{r}21 \\
12 \\
0\end{array}$ & $\begin{array}{c}20(30) \\
14(16) \\
\pm\end{array}$ & $\begin{array}{c}18(24) \\
8(16) \\
0\end{array}$ & $\begin{array}{c}12(20) \\
(13) \\
0\end{array}$ & $\begin{array}{c}(31) \\
(18) \\
0\end{array}$ & $\begin{array}{c}(28) \\
(15) \\
0\end{array}$ & $\begin{array}{c}30(33) \\
16(17) \\
0\end{array}$ & $\begin{array}{l}\text { N.G. } \\
\text { N.G. } \\
\text { N.G. }\end{array}$ & $\begin{array}{r}40 \\
28 \\
0\end{array}$ & $\begin{array}{r}17 \\
10 \\
0\end{array}$ & $\begin{array}{l}14 \\
\pm \\
0\end{array}$ & $\begin{array}{c}14 \\
\pm \\
0\end{array}$ \\
\hline 5 & $\begin{array}{l}10 \\
1 \\
0.1\end{array}$ & $\begin{array}{l} \pm \\
0 \\
0\end{array}$ & $\begin{array}{l}0 \\
0 \\
0\end{array}$ & $\begin{array}{l}\text { N.D. } \\
\text { N.D. } \\
\text { N.D. }\end{array}$ & $\begin{array}{l}\text { N.D. } \\
\text { N.D. } \\
\text { N.D. }\end{array}$ & $\begin{array}{l}\text { N.D. } \\
\text { N.D. } \\
\text { N.D. }\end{array}$ & $\begin{array}{l}\text { N.D. } \\
\text { N.D. } \\
\text { N.D. }\end{array}$ & $\begin{array}{l}\text { N.D. } \\
\text { N.D. } \\
\text { N.D. }\end{array}$ & $\begin{array}{l}\text { N.D. } \\
\text { N.D. } \\
\text { N.D. }\end{array}$ & $\begin{array}{l}\text { N.D. } \\
\text { N.D. } \\
\text { N.D. }\end{array}$ & $\begin{array}{l}\text { N.D. } \\
\text { N.D. } \\
\text { N.D. }\end{array}$ & $\begin{array}{l}\text { N.D. } \\
\text { N.D. } \\
\text { N.D. }\end{array}$ & $\begin{array}{l}\text { N.D. } \\
\text { N.D. } \\
\text { N.D. }\end{array}$ \\
\hline $\begin{array}{l}\text { Omadine } \\
\text { sodium }\end{array}$ & $\begin{array}{l}10 \\
1 \\
0.1\end{array}$ & $\begin{array}{l}30 \\
25 \\
16\end{array}$ & $\begin{array}{c}22(26) \\
15(18) \\
\pm\end{array}$ & $\begin{array}{c}20(25) \\
10(16) \\
(10)\end{array}$ & $\begin{array}{l}18 \\
12 \\
\pm\end{array}$ & $\begin{array}{c}15(40) \\
27(29) \\
0\end{array}$ & $\begin{array}{c}15(41) \\
(27) \\
(9)\end{array}$ & $\begin{array}{l}32(46) \\
25(38) \\
13(16)\end{array}$ & $\begin{array}{l}\text { N.G. } \\
\text { N.G. } \\
\text { N.G. }\end{array}$ & $\begin{array}{c}\text { N.G. } \\
40 \\
0\end{array}$ & $\begin{array}{l}28 \\
20 \\
10\end{array}$ & $\begin{array}{r}24 \\
12 \\
0\end{array}$ & $\begin{array}{r}22 \\
13 \\
0\end{array}$ \\
\hline Nystatin & $\begin{array}{l}10 \\
1 \\
0.1\end{array}$ & $\begin{array}{c}19 \\
(12) \\
0\end{array}$ & $\begin{array}{c}19 \\
(12) \\
0\end{array}$ & $\begin{array}{c}18 \\
(8) \\
0\end{array}$ & $\begin{array}{l}14 \\
(8)\end{array}$ & $\begin{array}{c}17(19) \\
8(10) \\
0\end{array}$ & $\begin{array}{c}16 \\
\pm \\
0\end{array}$ & $\begin{array}{r}19 \\
8 \\
0\end{array}$ & $\begin{array}{l}\text { N.G. } \\
\text { N.G. } \\
\text { N.G. }\end{array}$ & $\begin{array}{l}\text { N.G. } \\
\text { N.G. } \\
\text { N.G. }\end{array}$ & $\begin{array}{r}15 \\
8 \\
0\end{array}$ & $\begin{array}{c}14 \\
(12) \\
0\end{array}$ & $\begin{array}{l}15 \\
\pm \\
0\end{array}$ \\
\hline $\begin{array}{l}\text { Ampho- } \\
\text { tericin B }\end{array}$ & $\begin{array}{l}10 \\
1 \\
0.1\end{array}$ & $\begin{array}{r}14 \\
7 \\
0\end{array}$ & $\begin{array}{r}15 \\
9 \\
0\end{array}$ & $\begin{array}{c}11 \\
\pm \\
0\end{array}$ & $\begin{array}{c}10 \\
\pm \\
0\end{array}$ & $\begin{array}{r}16 \\
9 \\
0\end{array}$ & $\begin{array}{c}12 \\
\pm \\
0\end{array}$ & $\begin{array}{r}23 \\
15 \\
0\end{array}$ & $\begin{array}{l}\text { N.G. } \\
\text { N.G. } \\
\text { N.G. }\end{array}$ & $\begin{array}{r}22 \\
9 \\
0\end{array}$ & $\begin{array}{c}12 \\
\pm \\
0\end{array}$ & $\begin{array}{c}13(20) \\
12(16) \\
0\end{array}$ & $\begin{array}{l}10 \\
\pm \\
0\end{array}$ \\
\hline
\end{tabular}

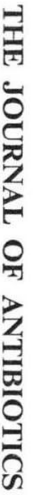

1. Sabouraud's glucose agar; 2. Trypticase soy agar; 3. Penassay seed agar; ( ) Hazy inhibition edges; \pm Very small inhibition zone; N.G. no growth;

N.D. not done 
Table 4. In vitro activity of 2-pyridinethiol 1-oxide cephalosporins and of controls against 15 bacterial strains

\begin{tabular}{|c|c|c|c|c|c|c|c|c|}
\hline \multirow{2}{*}{ Organism } & \multicolumn{8}{|c|}{ Minimal inhibitory concentrations $(\mu \mathrm{g} / \mathrm{ml})$} \\
\hline & 1 & 2 & 3 & 4 & 5 & Omadine & Cefazolin & Cephalexin \\
\hline Staph. aureus HH 127 & 3.1 & 1.6 & 1.6 & 3.1 & 0.4 & 3.1 & 0.4 & 6.3 \\
\hline $\begin{array}{l}\text { Staph. aureus SK\&F } \\
23390\end{array}$ & 1.6 & 0.4 & 0.8 & 1.6 & 0.2 & 3.1 & 0.2 & 3.1 \\
\hline $\begin{array}{l}\text { Staph. aureus Villaluz } \\
\text { (M. R.) SK\&F } 70399\end{array}$ & 12.5 & 12.5 & 3.1 & 3.1 & 50 & 1.6 & 25 & 50 \\
\hline Strep. faecalis $\mathrm{HH} 34358$ & 50 & 50 & 12.5 & 25 & 25 & 6.3 & 12.5 & 50 \\
\hline E. coli $\mathrm{SK} \& \mathrm{~F} 12140$ & 3.1 & 1.6 & 3.1 & 12.5 & 1.6 & 12.5 & 1.6 & 6.3 \\
\hline E. coli $\mathrm{HH} 33779$ & 12.5 & 3.1 & 6.3 & 12.5 & 1.6 & 12.5 & 1.6 & 12.5 \\
\hline $\begin{array}{l}\text { Kl. pneumoniae } \\
\text { SK\&F } 4200\end{array}$ & 3.1 & 1.6 & 3.1 & 6.3 & 0.8 & 6.3 & 1.6 & 6.3 \\
\hline $\begin{array}{l}\text { Kl. pneumoniae } \\
\text { SK\&F } 1200\end{array}$ & 0.8 & 1.6 & 6.3 & 6.3 & 0.8 & 12.5 & 0.8 & 6.3 \\
\hline $\begin{array}{l}\text { Salmonella paratyphi } \\
\text { ATCC } 12176\end{array}$ & 3.1 & 1.6 & 3.1 & 6.3 & 0.2 & 12.5 & 1.6 & 3.1 \\
\hline $\begin{array}{l}\text { Shigella paradysenieriae } \\
\text { HH } 117\end{array}$ & 1.6 & 0.8 & 1.6 & 3.1 & 0.4 & 6.3 & 3.1 & 12.5 \\
\hline Ps. aeruginosa $\mathrm{HH} 63$ & $>200$ & $>200$ & $>200$ & $>200$ & $>200$ & 200 & $>200$ & $>200$ \\
\hline $\begin{array}{l}\text { Serratia marcescens } \\
\text { ATCC } 13880\end{array}$ & 200 & 25 & 50 & 50 & $>200$ & 25 & $>200$ & 50 \\
\hline Proteus morganii 179 & 100 & 6.3 & 25 & 25 & 200 & 12.5 & $>200$ & $>200$ \\
\hline $\begin{array}{l}\text { Enterobacter aerogenes } \\
\quad \text { ATCC } 13048\end{array}$ & 12.5 & 3.1 & 3.1 & 6.3 & 3.1 & 12.5 & 12.5 & 12.5 \\
\hline $\begin{array}{l}\text { Enterobacter cloacae } \\
\text { HH } 31254\end{array}$ & 6.3 & 1.6 & 3.1 & 6.3 & 1.6 & 12.5 & 3.1 & 6.3 \\
\hline
\end{tabular}

Table 5. Efficacy $\left(\mathrm{ED}_{50}\right)$ of subcutaneously and orally administered 2-pyridinethiol 1-oxide cephalosporins

\begin{tabular}{|c|c|c|c|c|c|c|}
\hline \multirow{3}{*}{$\begin{array}{c}\text { SK\&F } \\
\text { Number or } \\
\text { compound }\end{array}$} & \multicolumn{3}{|c|}{ E. coli $\sharp 12140$} & \multicolumn{3}{|c|}{ K. pneumoniae $\sharp 4200$} \\
\hline & \multirow{2}{*}{$\begin{array}{l}\mathrm{MIC} \\
\mu \mathrm{g} / \mathrm{ml}\end{array}$} & \multicolumn{2}{|c|}{$\mathrm{ED}_{50}(\mathrm{mg} / \mathrm{kg})$} & \multirow{2}{*}{$\begin{array}{c}\mathrm{MIC} \\
\mu \mathrm{g} / \mathrm{ml}\end{array}$} & \multicolumn{2}{|c|}{$\mathrm{ED}_{50}(\mathrm{mg} / \mathrm{kg})$} \\
\hline & & Subcut. & Oral & & Subcut. & Oral \\
\hline 1 & 3.1 & 44 & $>200$ & 3.1 & 100 & $>200$ \\
\hline 2 & 1.6 & 9.4 & 100 & 1.6 & 3 & 100 \\
\hline 3 & 3.1 & 9.4 & $>50 *$ & 3.1 & 14 & $>50 *$ \\
\hline 4 & 12.5 & 6.2 & 50 & 6.3 & 5 & 35 \\
\hline 5 & 1.6 & 4 & $>50^{*}$ & 0.8 & 9.5 & N.T. \\
\hline Omadine & 12.5 & $>200$ & N.T. & 6.3 & N.T. & N.T. \\
\hline
\end{tabular}

* Highest level tested. N.T. Not tested

data. Peak serum concentrations in mice ( $20 \mathrm{mg} / \mathrm{kg}$ subcutaneously) were 27,11 and $24 \mu \mathrm{g} / \mathrm{ml}$ for compounds 1, 3 and 4, respectively. Oral administration of the phenylglycine analogs resulted in only trace serum levels and low urinary excretion, although they protected mice at high dose levels. This phenomenon has been observed in our laboratory with other phenylglycine cephalosporins.

\section{Discussion}

GotTSTEIN et al. reported that N-benzyldithiocarbamoylacetamido-cephalosporanic acid had in vitro antifungal activity ${ }^{4}$. Additional semisynthetic cephalosporins with in vitro antifungal activities are described here. The antifungal activity of one of these cephalosporins (compound 2) previously has been reported by O'CALLAGHAN et al ${ }^{9}$. These cephalosporins incorporate a 2-pyridinethiol 1-oxide 
moiety at the $3^{\prime}$-position. Antifungal activity was not observed when this moiety is a part of the 7substituent. The cephalosporin described by GotTsTEIN et al..$^{4)}$ is a dithiocarbamate and thus might be expected to have antifungal activity per se. O'CALLAGHAN et al..$^{8,9)}$ have demonstrated that one of the cephalosporins described here (compound 2) releases omadine (an antifungal agent in itself) under conditions of $\beta$-lactam ring opening. The data of GreENwood and O'Grady support this finding ${ }^{5)}$. Our data also tends to support this assumption with the exception of the activity versus $C$. albicans where the antifungal activities of the cephalosporins are equal to or greater than that of omadine (Table 2). The antimicrobial activity of omadine is due to its chelate-formation with cations ${ }^{2}$. With copper, it immediately forms a greenish-brown water-insoluble complex. None of the cephalosporins with this side chain showed complex formation upon the addition of copper sulfate. Although this would tend to suggest that omadine was not released from the molecule, the study was not carried out under the in vitro antifungal test conditions. In any case, neither of these cephalosporin types are active against Candida infections in laboratory animals. In the case of these cephalosporins, it may be that the antifungal agent, whatever it is, may not reach high enough concentration at the site of infection. This is especially likely if omadine is the sole active factor as it failed to protect mice infected with $C$. albicans. Although the intact cephalosporins were capable of producing protective antibacterial blood levels in mice, they failed to protect against $C$. albicans infection as did omadine itself. The 2-pyridinethiol 1oxide side chain is still of some research interest in that this grouping has been attached at the $3^{\prime}$-position to a cephamycin nucleus; however, no antifungal data was reported with this compound ${ }^{10}$. Other reports of similar 1-oxide side chains attached to a $\beta$-lactam nucleus are available but no biological data has been reported for these compounds ${ }^{3)}$.

\section{Acknowledgments}

The authors wish to express their thanks to Drs. J. R. E. Hoover and G. L. DunN for their assistance in preparation of this manuscript and members of the Medicinal Chemistry Staff, SK\&F Laboratories, who synthesized these cephalosporins. We also acknowledge the technical help of J. R. GUARINI and C. S. SACHS.

\section{References}

1) Actor, P.; J. V. Uri, L. Phillips, C. S. Sachs, J. R. Guarini, I. Zajac, D. A. Berges, G. L. Dunn, J. R. E. Hoover \& J. A. WeISBACH: Laboratory studies with cefatrizine (SK\&F 60771) a new broad-spectrum orally active cephalosporin. J. Antibiotics 28: 594 601, 1975

2) Albert, A.: Selective toxicity. pp. 340 341. Methuen \& Co. Ltd., London, 1968

3) Edwards, M. L.; R. E. Bambury \& H. W. Ritter: $\quad \beta$-Lactam antibiotics with N-oxide side chains. 1 Quinoxaline N-oxides. J. Med. Chem. 19: 330 333, 1976

4) Gottstein, W. J.; A. H. Eachus, P. F. Misco, L. C. Cheney, M. Misiek \& K. E. Price: $\beta$-Lactam antimicrobial agents which possess antifungal activity. J. Med. Chem. 14: 770 772, 1971

5) Greenwood, D. \& F. O'Grady: Dual-action cephalosporin utilizing a novel therapeutic principle. Antimicr. Agents \& Chemoth. 10: 249 252, 1976

6) Litchfield, J. T., Jr. \& F. Wilcoxon: A simplified method of evaluating dose-effect experiments. J. Pharmacol. Exp. Ther. 96: 99 113, 1949

7) Lukens, R. J.: Chemistry of fungicidal action. p. 104, Springer-Verlag, New York, Heidelberg, Berlin, 1971

8) O'Callaghan, C. H.; S. M. Kirby, A. Morris, R. E. Waller \& R. E. Duncombe: Correlation between hydrolysis of the $\beta$-lactam bond of the cephalosporin nucleus and expulsion of the 3-substituent. J. Bacteriol. 110: 988 991, 1972

9) O'Callaghan, C. H.; R. B. Sykes \& S. E. Stanforth: A new cephalosporin with a dual mode of action. Antimicr. Agents \& Chemoth. 10: $245 \sim 248,1976$

10) Shimizu, B.; M. Kaneko, M. Kimura \& S. Sugawara: Synthesis of 7 $\alpha$-methoxy-7-[2-(substituted thio) acetamido]cephalosporin derivatives and their antibacterial activities. Chem. Pharm. Bull. 24: 2629 2636, 1976

11) Uri, J. V.; P. Actor, J. R. Guarini, L. Phillips, D. Pitkin, R. M. DeMarinis \& J. A. Weisbach: Biological and chemotherapeutic studies on three semisynthetic cephamycins. J. Antibiotics 31: 82 91, 1978 